\title{
Decanoylcarnitine Measurement
}

National Cancer Institute

\section{Source}

National Cancer Institute. Decanoylcarnitine Measurement. NCI Thesaurus. Code C156536.

The determination of the amount of decanoylcarnitine present in a sample. 